\title{
Resveratrol Prevents Cognitive Impairment in Type 2 Diabetic Mice by Upregulating Nrf2 Expression and Transcriptional Level
}

This article was published in the following Dove Press journal: Diabetes, Metabolic Syndrome and Obesity: Targets and Therapy

\author{
Xiaoxiao Wang $\mathbb{1}^{\prime}$ \\ Hui Fang ${ }^{2}$ \\ Gang $X u^{3}$ \\ Ying Yang ${ }^{2}$ \\ Ruizhe $\mathrm{Xu}^{4}$ \\ Qiang Liu $^{5}$ \\ Xiangyu Xue $\mathrm{X}^{5}$ \\ Jiaqi Liu $^{5}$ \\ Hezhi Wang (1D ${ }^{6}$
}

'Department of Internal Medicine, Hebei Medical University, Shijiazhuang, Hebei 0500 17, People's Republic of China;

${ }^{2}$ Second Department of Endocrinology, Tangshan Gongren Hospital, Tangshan, Hebei 063000, People's Republic of China; ${ }^{3}$ Department of Burns and Orthopedics, Tangshan Gongren Hospital, Tangshan, Hebei 063000, People's Republic of China; ${ }^{4}$ Department of Clinical Medicine, Tangshan Vocational and Technical College, Tangshan, Hebei, 063000, People's Republic of China; ${ }^{5}$ Department of Internal Medicine, North China University of Science and Technology, Tangshan, Hebei 063000, People's Republic of China; ${ }^{6}$ Department of Surgery, Hebei Medical University, Shijiazhuang 050017, People's Republic of China
Correspondence: Hui Fang Second Department of Endocrinology, Tangshan Gongren Hospital, 27 Wenhua Road, Lubei District, Tangshan, Hebei 063000, People's Republic of China

Tel +86- I383|58|838

$\mathrm{Fax}+00863152814801$

Email fanghui2018@I26.com
Purpose: This study aimed to determine whether the natural antioxidant resveratrol (RSV) prevents type 2 diabetes mellitus (T2DM)-induced cognitive impairment and to explore whether redox-associated factor nuclear factor erythroid 2-related factor 2 (Nrf2) plays a critical role in the neuroprotective effect of RSV.

Materials and Methods: We established a T2DM model with 8-week-old male ICR mice by administration of a high-fat diet for 2 months and low-dose streptozotocin for 3 days. Then, diabetic and age-matched control mice were treated with or without RSV for 4 months every other day and subjected to the Morris water maze test. After the mice were euthanized, whole brains were sectioned for Nissl staining and immunofluorescence labeling. Hippocampal sections were observed by transmission electron microscopy to evaluate the ultrastructure of synapses. Inflammatory factors, oxidative stress-related indexes, and Nrf2 and downstream target gene expression were analyzed in hippocampal tissues by quantitative real-time PCR, Western blotting, and associated quantitative kits.

Results: In the Morris water maze test, compared to control mice, T2DM mice showed learning and memory impairments, but RSV treatment prevented the learning and memory decline in T2DM mice. Similarly, RSV prevented T2DM-induced hippocampal neuron destruction and synaptic ultrastructural damage. The expression levels of inflammatory factors and oxidative stress-related indicators were increased in the T2DM group compared with the control group but were decreased significantly by RSV treatment in the T2DM group. Additionally, the expression of $\mathrm{Nrf} 2$ and its downstream target genes was decreased in the T2DM group compared with the control group and was significantly increased by RSV treatment in the T2DM group.

Conclusion: RSV prevented T2DM-induced cognitive impairment through anti-inflammatory and antioxidant activities. This effect was accompanied by the upregulation of Nrf2 transcriptional activity and the increased expression of downstream antioxidant genes.

Keywords: Nrf2 activator, natural antioxidant, oxidative stress, inflammation

\section{Introduction}

Diabetes mellitus (DM) is a severe metabolic disease, and the increasing incidence of DM-related complications and mortality has aroused considerable public concern. According to epidemiological surveys, there were 382 million diabetic patients in $2013,90 \%$ of whom had type 2 diabetes mellitus (T2DM); the number of people with diabetes is anticipated to reach 592 million in $2035 .{ }^{1}$ T2DM causes many serious complications, including cardiovascular disease, retinopathy, kidney disease, and cognitive impairment. ${ }^{2}$ Consequently, treatments that effectively prevent and delay DM 
complications are urgently needed. Studies have shown that T2DM patients develop a significant decline in cognitive function, and of these patients, approximately 70\% eventually develop Alzheimer's disease; the risk of Alzheimer's disease in patients with T2DM is $1.5-2.0$ times that in the non-DM population. ${ }^{3,4}$ The hippocampus is a brain region associated with cognitive impairment and is one of the regions most sensitive to blood glucose fluctuations. ${ }^{5}$ Our team previously successfully furthered our understanding of the mechanisms of DM-associated cognitive impairment. ${ }^{6-8}$ Inflammation and oxidative stress are involved in the pathogenesis of cognitive decline, and a vicious circle forms between these two factors to further accelerate brain damage and cognitive impairment. ${ }^{9,10}$ Therefore, high blood glucose, inflammation and oxidative stress may result in a greater vulnerability to cognitive impairment. Oxidative stress is produced in vivo when excess reactive oxygen species (ROS) overwhelm the defense of endogenous antioxidants. Thus, enhancing the antioxidant ability of the hippocampus may be a prospective, valuable method for preventing cognitive impairment in T2DM.

Nuclear factor erythrocyte 2-related factor 2 (Nrf2) acts as a redox-sensitive transcription factor in oxidative stress and inflammation-related tissue damage. Under physiological conditions, the combination of Nrf2 and Kelch-like ECHassociated protein 1 (Keap1) in the cytoplasm causes Nrf2 to undergo ubiquitination and degradation; therefore, the basal expression of $\mathrm{Nrf} 2$ and downstream targets are necessary to maintain cell homeostasis. ${ }^{11} \mathrm{Nrf} 2$ is activated and dissociated from Keap1 after stimulation by external oxidative stress molecules or nucleophiles. Then, it enters the nucleus to initiate antioxidant response elements (AREs) to regulate downstream antioxidant targets, such as heme oxygenase-1 (HO-1) and $\mathrm{NAD}(\mathrm{P}) \mathrm{H}$ : quinone oxidoreductase (NQO1), metallothionein (MT), superoxide dismutase (SOD) and catalase (CAT). Eventually, all antioxidant targets neutralize excess ROS to maintain cellular redox balance and provide a cytoprotective function. ${ }^{12}$ In vivo experiments have shown that Nrf2 knockout in amyloid precursor protein (APP)/presenilin1 (PS1) mice exacerbate the spatial learning and memory deficits. ${ }^{13}$ Furthermore, in vitro experiments have shown that activation of the $N r f 2$ gene can reverse the excessive production of ROS in high glucose-induced neuroblastoma cells (SH-SY5Y) and significantly improve cell viability. ${ }^{14}$

Resveratrol (RSV), a phenolic compound, is a natural antioxidant present at high levels in grapes and Polygonum cuspidatum. Previous studies have proven the extensive biological and pharmacological properties of RSV, such as anti- inflammatory, antioxidative, and antiapoptotic properties. ${ }^{15-17}$ The results showed that RSV induced excellent diabetes resistance in 2-10 weeks, primarily through a reduction in inflammatory cytokines, lipid peroxidation, and apoptosis, while also significantly enhancing antioxidant defense effects. ${ }^{18-21} \mathrm{RSV}$ acts as a neuroprotective agent by reducing the level of the oxidative stress marker malondialdehyde (MDA) in the hippocampus of rats with vascular dementia. ${ }^{22}$ However, whether RSV can prevent T2DM-induced cognitive impairment remains unclear.

Therefore, we induced T2DM in ICR male mice to study whether RSV can prevent T2DM-induced cognitive dysfunction and determine the mechanism of action by analyzing hippocampal inflammation, oxidative stress, and Nrf2 and downstream antioxidant gene expression.

\section{Materials and Methods}

\section{Animal and Experimental Design}

All experimental animal protocols were approved by the Animal Ethics Committee of the Hebei General Hospital and complied with the "International Laboratory Animal Management Regulations." Animals were raised in the animal barrier system of the clinical research center of Hebei General Hospital. The temperature was controlled at approximately $24{ }^{\circ} \mathrm{C}$ with a 12 -hour light/dark cycle. We selected 8-week-old male ICR mice (Vital River Laboratory Animal Technology Co. Ltd., Beijing, China) weighing approximately $25-30 \mathrm{~g}$ as the research subjects. All mice were adapted to the feed under the above conditions for one week before each experiment. The normal diet (ND) (no. $12450 \mathrm{~J}$ ) contained $10 \% \mathrm{kcal}$ fat, and the high-fat diet (HFD) contained 60\% kcal fat (no. 12492; Research Diets, NJ, USA). The pathogenesis of T2DM has two aspects: insulin resistance and insufficient insulin secretion. ${ }^{23}$ After 2 months of HFD feeding, an intraperitoneal glucose tolerance test (IPGTT) was performed on the mice to verify the successful induction of insulin resistance (Figure 1A). By calculating the area under the curve (AUC) of blood glucose, it was found that the AUC of the HFD group was significantly higher than that of the ND group (Figure 1B). In some obese mice with insulin resistance, T2DM was induced by administration of low-dose streptozotocin (STZ) after 6 hours of fasting for three days. STZ (Enzo, NYC, USA) was dissolved in $0.1 \mathrm{~mol} / \mathrm{L}$ sodium citrate buffer at a $\mathrm{pH}$ of 4.4 and immediately protected from light and injected. STZ $(50 \mathrm{mg} / \mathrm{kg})$ was administered by repeated intraperitoneal injection for 3 days, while the control group 
A

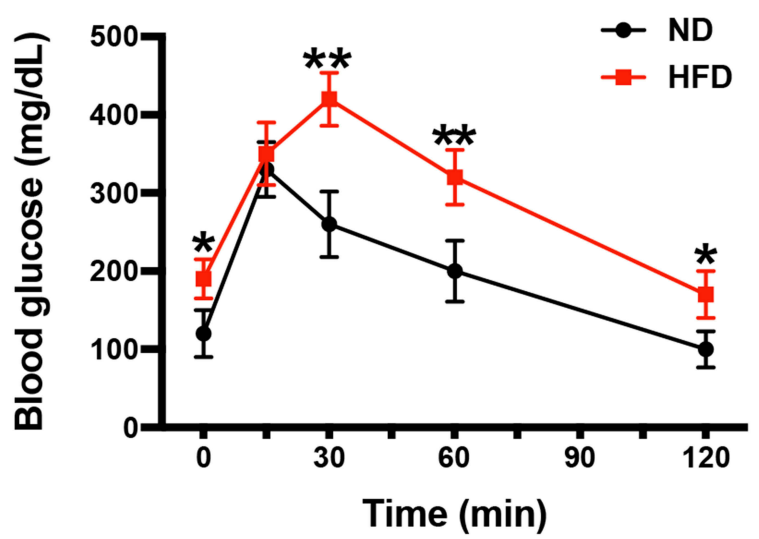

B

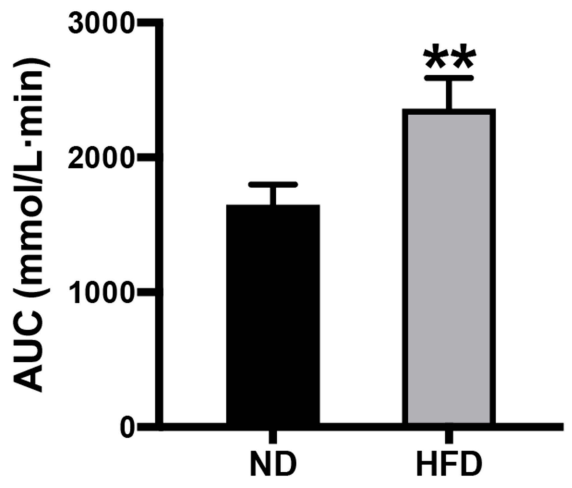

C

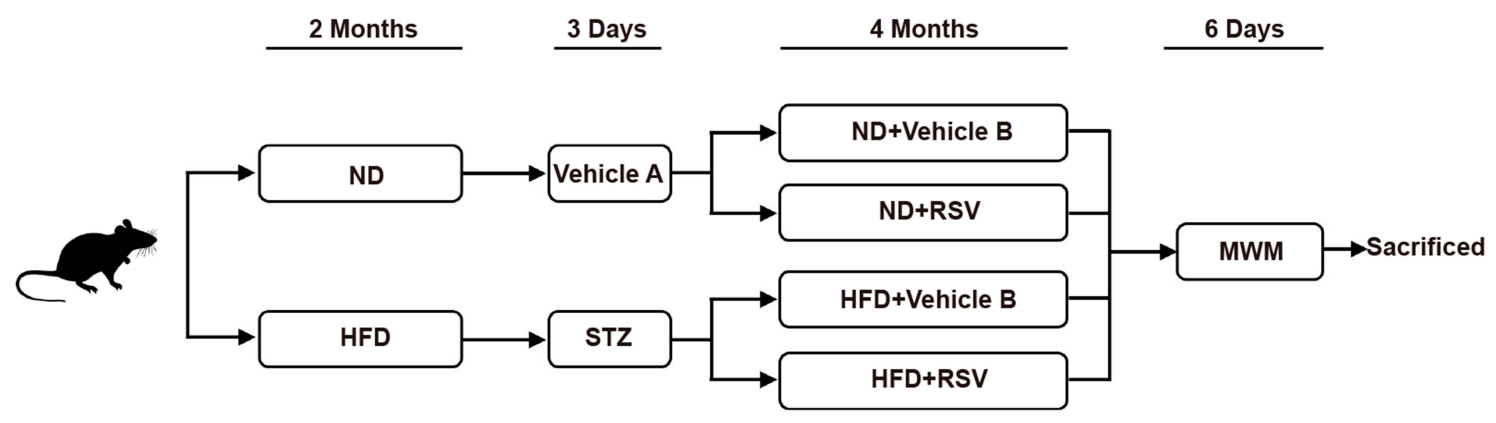

D

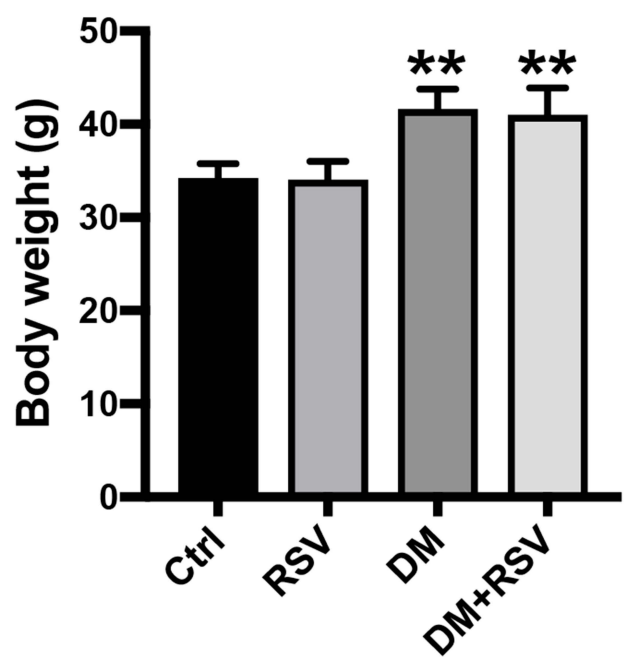

E

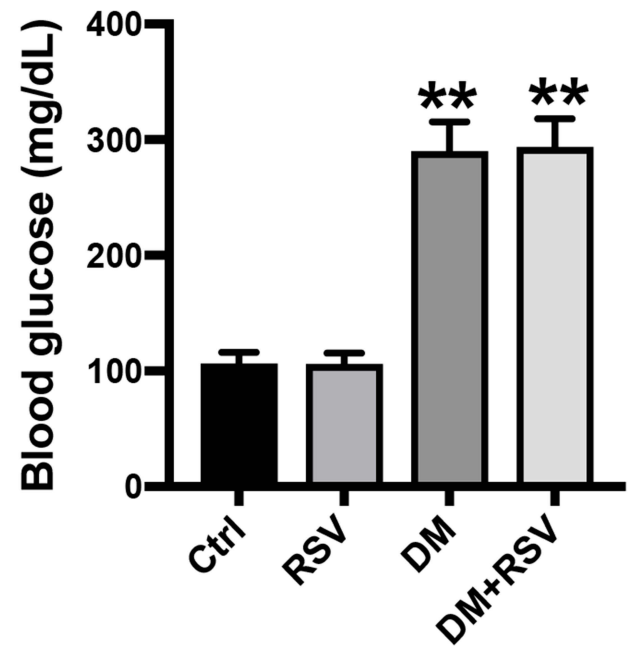

Figure I Experimental design and intergroup metabolic parameter comparison. (A) IPGTT test two months after HFD induction. (B) The AUC of blood glucose in the IPGTT test. The data are expressed as the mean $\pm S D(n=30)$. $* P<0.05$, $* * P<0.01$ vs ND. (C) Schematic of the experimental process. (D, E) After 6 months of intervention in the mice, the body weights of the mice in the four groups were measured $(\mathbf{D})$, and blood glucose levels were analyzed $(E)$. The data are expressed as the mean \pm SD $(n=I 5)$. $* * P<0.0 I$ vs $C$ trl. Abbreviations: IPGTT, intraperitoneal glucose tolerance test; HFD, high-fat diet; AUC, area under the curve.

was intraperitoneally injected with an equal amount of citrate buffer (vehicle A). Five days after the administration of STZ, the glucose level was measured in tail vein blood collected from mice that had been fasted for 3 hours. Mice with blood glucose $\geq 250 \mathrm{mg} / \mathrm{dl}(13.9 \mathrm{mmol} / \mathrm{L})$ were defined as T2DM model mice. Mice were fed RSV 
(Sigma Aldrich, St. Louis, MO, USA) in $0.5 \%$ sodium carboxymethylcellulose by intragastric administration at $30 \mathrm{mg} / \mathrm{kg}$ every other day for 4 months, and the control mice were given the same amount of $0.5 \%$ carboxymethylcellulose (vehicle B). The mice continued to be fed either the HFD or ND during the RSV intervention. We defined four experimental groups: ND + vehicle B (control), ND + RSV (RSV), HFD/STZ + vehicle B (DM), and HFD/STZ + RSV (DM+RSV). The mice were tested for the Morris water maze (MWM) after RSV intervention for 4 months and euthanized the next day, the brain and hippocampus were collected for subsequent experiments (Figure 1C).

The RSV dose of $30 \mathrm{mg} / \mathrm{kg}$ was chosen because of the following three factors. (1) RSV has poor water solubility and rapid metabolism; the plasma concentration can reach the peak in one hour, and only a small amount reaches the target organ, resulting in poor bioavailability. ${ }^{24,25}$ Because of these characteristics of RSV, the current dose of RSV used in mice research is most often $10 \mathrm{mg} / \mathrm{kg}-40 \mathrm{mg} / \mathrm{kg}$. ${ }^{26,27}$ (2) Although RSV seems to have good tolerance and safety, a higher oral dose does not improve the therapeutic effect. ${ }^{28}$ (3) Because of the experimental design of the treatment of chronic diseases, we chose $30 \mathrm{mg} / \mathrm{kg}$ every other day for 4 months to ensure the maximum efficacy while reducing the incidence of side effects.

\section{Morris Water Maze (MWM)}

The spatial learning and memory ability of mice was determined through an MWM test. ${ }^{29}$ The maze was black and divided into four quadrants, with a platform placed in the third quadrant. The maze was filled with water to $1.5 \mathrm{~cm}$ above the platform, and different shapes and colors were attached to the labyrinth wall. Each training session began by placing the mice into the water in any of the other three quadrants not containing the platform and ended when the mice climbed onto the platform. If the mice failed to find the platform within 60 seconds, the escape latency was recorded as 60 seconds, and the mice were carefully guided onto the platform, where it remained for 15 seconds. The mice were then dried and returned to the home cage. The mice were tested in the hidden platform test 4 times a day for 5 consecutive days during the training period to evaluate learning ability. The platform was removed on the sixth day of training, and the mice were placed in the maze again to test spatial memory. For the spatial memory ability test, the mice were placed in the water and allowed to swim for 60 seconds, and spatial memory was evaluated by the amount of time the mice spent in the target quadrant and the number of times the mice traversed the previous platform location. The swimming speed was also calculated to control for the effect of motor function on the outcome. A camera mounted above the maze was used to record swimming trajectories and record test data. An IBM computer (Armonk, NY, USA) with a tracking program was attached to the ceiling camera for the collection and analysis of the data.

\section{Nissl Staining}

Mice were perfused with saline and paraformaldehyde. Then, the entire brain was removed and placed in paraformaldehyde fixative. After fixation for more than 24 hours, the brain was embedded in paraffin and sliced. Paraffin sections were dewaxed and stained with toluidine blue. Three sections were randomly selected from each mouse, and the number of neurons in the hippocampal CA1 region was counted at high magnification $(400 \times)$ under a light microscope (Nikon, Tokyo, Japan) and photographed.

\section{Transmission Electron Microscopy (TEM)}

The mice were anesthetized and then perfused with physiological saline and $2.5 \%$ glutaraldehyde. The hippocampal CA1 area was rapidly separated, cut into $1-\mathrm{mm}^{3}$ tissue pieces and immersed in $2.5 \%$ glutaraldehyde at $4{ }^{\circ} \mathrm{C}$ overnight. After the sample was rinsed three times in 0.1 M phosphate buffer, it was fixed in a $1 \%$ osmium acid fixative for 3 hours and then dehydrated with an ascending gradient of acetone. Pure acetone and epoxy resin Epon 812 were used for infiltration and embedding, and the tissue was then sliced into ultrathin slices of $50 \mathrm{~nm}$ and stained with $3 \%$ uranium acetate and lead citrate. Finally, the ultrastructure of synapses was observed and photographed by TEM (CM-120, Philips, Amsterdam, Netherlands).

\section{Western Blot Analysis}

Hippocampal tissue was placed in RIPA lysis buffer containing a phosphatase inhibitor and a protease inhibitor $(1 \mathrm{mg}$ : $10 \mu \mathrm{L}$ ) and homogenized by a grinder. Hippocampal cell cytoplasm and nuclei were isolated for nuclear protein analysis using a nuclear protein extraction kit (BestBio, Shanghai, China). The homogenized protein was collected by centrifugation at $12,000 \mathrm{x}$ for 20 minutes at $4{ }^{\circ} \mathrm{C}$, and the protein concentration was measured by a BCA Protein Assay Kit (Thermo Scientific, Barrington, IL, USA). Protein samples were separated by $10-12 \%$ SDS-PAGE and electrotransferred onto a polyvinylidene fluoride membrane. The PVDF membrane was blocked with $5 \%$ skim 
milk powder for 2 hours and incubated overnight at $4{ }^{\circ} \mathrm{C}$ with the following antibodies: anti- $\beta$-actin (1:5000, Abcam, Cambridge, MA, USA), anti-Histone H3 (1:3000, Abcam, Cambridge, MA, USA), anti-Nrf2, anti-tumor necrosis factor- $\alpha(\mathrm{TNF}-\alpha)$, anti-interleukin-1 $\beta$ (IL-1 $\beta)$, anti-3-nitrotyrosine (3-NT), anti-MT (1:1000, Abcam, Cambridge, MA, USA), anti-HO-1 (1:2000 Abcam, Cambridge, MA, USA), and anti-NQO1 (1:10,000, Abcam, Cambridge, MA, USA). The membrane was washed with Tris-HCl buffer containing Tween 20 and incubated with horseradish peroxidase (HRP)-labeled antibody for 50 minutes at room temperature. The target band was visualized in the imaging system by the ultrasensitive enhanced chemiluminescence substrate (Thermo Scientific, Waltham, MA, USA). Quantitative densitometry and analysis of the recognized bands were performed using ImageJ.

\section{Quantitative Real-Time PCR}

According to the manufacturer's instructions, total RNA was extracted from the hippocampus using a total RNA Extraction Kit (Tiangen, Beijing, China). RNA concentration and purity were quantified by a Nanodrop 2000 spectrophotometer. Total RNA was synthesized into complementary DNA (cDNA) using an RNA reverse transcription kit (Takara, Beijing, China). PCR buffers were prepared using the SYBR Premix Ex Taq II kit (Takara, Beijing, China) following the instructions. Each sample was amplified in triplicate using the ABI 7500 Real-Time PCR System. Invitrogen Biotech Company synthesized the primers shown in Table 1. The expression of each target gene was calculated according to the $2-\Delta \Delta \mathrm{CT}$ method, and the results are shown as the changes in mRNA expression of the target gene relative to that of $\beta$-actin.

\section{Immunofluorescence Staining}

Paraffin sections of the whole brains of mice were prepared according to the Nissl staining method described above. The dewaxed tissue sections were repaired in a microwave oven with EDTA antigen repair buffer $(\mathrm{pH}$ 8.0) and then incubated with bovine serum albumin for 30 minutes. The sections were incubated with the following antibodies overnight at $4{ }^{\circ} \mathrm{C}$ : anti-8 hydroxyguanosine (8-OHdG, 1:200, Bios, Beijing, China), anti-glial fibrillary acidic protein (GFAP, 1:1000, Abcam, Cambridge, MA), and anti-Nrf2 (1:200, Abcam, Cambridge, MA). The glass slides were incubated with the corresponding secondary antibodies at room temperature for 50 minutes. The images were observed under a fluorescence microscope after incubation with 4,6-diamidino-2-phenylindole (DAPI) at room temperature for 10 minutes.

\section{Oxidative Stress Indicator Assay}

The levels of MDA (A003-1), SOD (A001-1), and CAT (A007-1) in hippocampal tissue were measured following the relevant quantitative kit instructions (Jiancheng, Nanjing, China).

\section{Statistical Analysis}

All data analyzed by blind collection was expressed as the mean $\pm \mathrm{SD}$, and statistical analysis was performed using GraphPad Prism Version 8.0 (GraphPad Software, CA, USA). The differences between the two groups were compared using the Student's $t$-test. Four groups were compared using one-way ANOVA and subsequent Tukey multiple comparison tests. The escape latency in the MWM was analyzed by two-way repeated-measures ANOVA and Bonferroni post hoc test. Statistical significance was considered to be $P<0.05$.

Table I Primers Used in Quantitative Real-Time PCR

\begin{tabular}{|l|l|l|}
\hline Gene & Forward Primer & Reverse Primer \\
\hline Nrf2 & 5'-AAGAATAAAGTCGCCGCCCA-3' & 5'-AGATACAAGGTGCTGAGCCG-3' \\
HO-I & 5'-ATGGCGTCACTTCGTCAGAG-3' & 5'-GCTGATCTGGGGTTTCCCTC-3' \\
NQOI & 5'-TAGCCTGTAGCCAGCCCTAA-3' & 5'-GCCTCCTTCATGGCGTAGTT-3' \\
MT & 5'-GCCTGCAAATGCAAACAATGC-3' & 5'-AGCTGCACTTGTCGGAAGC-3' \\
$\beta$-actin & 5'-CATCACTATCGGCAATGAGC-3' & 5'-GACAGCACTGTGTTGGCATA-3' \\
\hline
\end{tabular}

Abbreviations: RSV, resveratrol; T2DM, type 2 diabetes mellitus; Nrf2, nuclear factor erythroid 2-related factor 2; DM, diabetes mellitus; AD, Alzheimer's disease; ROS, reactive oxygen species; Nrf2, nuclear factor erythroid 2-related factor 2; Keapl, Kelch-like $\mathrm{ECH}$-associated protein I; AREs, antioxidant response elements; HO-I, heme oxygenase-I; NQOI, NAD(P)H: quinone oxidoreductase; MT, metallothionein; SOD, superoxide dismutase; CAT, catalase; MDA, malondialdehyde; ND, normal diet; HFD, high-fat diet; STZ, streptozotocin; MWM, Morris water maze; TEM, Transmission electron microscopy; TNF- $\alpha$, tumor necrosis factor- $\alpha$; IL-I $\beta$, interleukin-I $\beta$; 3-NT, 3-nitrotyrosine; 8-OHdG, 8-hydroxyguanosine. 


\section{Results}

RSV Prevents T2DM-Induced Learning

\section{and Memory Impairment}

After 6 hours of fasting, the body weight and blood glucose of diabetic mice increased significantly, and there was no significant difference between the RSV-treated and untreated groups (Figure 1D and E).

In the MWM test, the escape latency was used to determine spatial information acquisition capabilities. The spatial memory of the mice was evaluated by removing the platform on the 6th day. Compared to the control group, the DM group exhibited a significantly increased escape latency in the MWM test on days 2-5. However, the escape latency was reduced considerably in the $\mathrm{DM}+\mathrm{RSV}$ group compared with the DM group on days 3-5 (Figure 2A). After the removal of the platform on day 6 , mice in the DM group crossed over the platform less frequently and remained in the platform quadrant for less time than mice in the control group. However, mice in the DM+RSV group crossed over the platform area more frequently and stayed longer in the platform quadrant than mice in the DM group (Figure 2B and C). There was no difference in swimming speed among the four groups, indicating that the experimental results were not affected by motor function (Figure 2C). These results suggest that compared to age-matched control mice, DM mice exhibit learning and memory impairment, but RSV treatment prevented the decline in learning and memory abilities in DM mice.

\section{RSV Prevents T2DM-Induced Hippocampal Neuronal Destruction and Synaptic Ultrastructural Damage}

To assess the effect of RSV on diabetes-associated cognitive impairment, we first used Nissl staining to analyze neuronal cell survival in this model. The number of neurons in the CA1 area of the hippocampus was significantly decreased, the gap was increased, and the volume was decreased in the DM group compared with the control group; moreover, the arrangement of neurons was irregular, and nuclear pyknosis was observed in the DM group. However, treatment with RSV prevented these pathological changes (Figure 3A). Synaptic structure and functional integrity are the physiological basis for information transmission between neurons. Therefore, TEM was used to observe changes in the synaptic morphology of the hippocampal CA1 region to reflect learning and memory ability. Compared with the synapses of control mice, the synapses of DM mice showed significant damage, such as blurred synaptic ultrastructure, synaptic cleft widening, fewer synaptic vesicles in the presynaptic membrane, and postsynaptic membrane thinning. However, there was no significant damage to the synaptic ultrastructure in the DM + RSV group (Figure 3B).

\section{RSV Prevents T2DM-Induced Hippocampal Inflammation}

Hippocampal inflammation was evaluated by the inflammatory factors TNF- $\alpha$ and IL-1 $\beta$. The Western blot results showed that the expression of TNF- $\alpha$ and IL-1 $\beta$ was significantly increased in the DM group compared with the control group. The expression level of these factors was significantly lower in the DM+RSV group than in the DM group (Figure 4AB and $\mathrm{B}$ ).

\section{RSV Prevents T2DM-Induced Hippocampal Oxidative Stress}

3-NT, 8-OHdG, and MDA levels were assessed to evaluate oxidative stress levels. We used Western blotting to detect 3-NT in the hippocampus as an indicator of protein nitrification. RSV treatment significantly prevented T2DM-induced hippocampal protein nitrification (3-NT) accumulation (Figure 5A). We used 8-OHdG and GFAP double immunofluorescence labeling to evaluate DNA oxidation in the neurons of the hippocampal CA1 region. The results showed that the expression of green 8-OHdG was significantly increased in the DM group compared with the control group. However, RSV treatment almost completely prevented T2DM-induced $8-\mathrm{OHdG}$ overexpression (Figure 5B). The lipid peroxidation product MDA in the DM group was significantly higher than that in the control group, and the content of MDA in the DM $+\mathrm{RSV}$ group was between that in the control group and that in the DM group (Figure 5C).

\section{RSV Upregulates Nrf2 Expression in the Hippocampus}

All the above experimental data indicated that RSV treatment might prevent T2DM-induced cognitive impairment. Therefore, we measured Nrf2 expression and nuclear accumulation in hippocampal neurons to explore whether RSV prevents this effect by upregulating Nrf2. The mRNA and protein expression levels of $\mathrm{Nrf} 2$ in the RSV group were significantly higher than those in the control group. In addition, RSV significantly prevented the decrease of Nrf2 expression in the DM group (Figure 6A and B). 


\section{A}

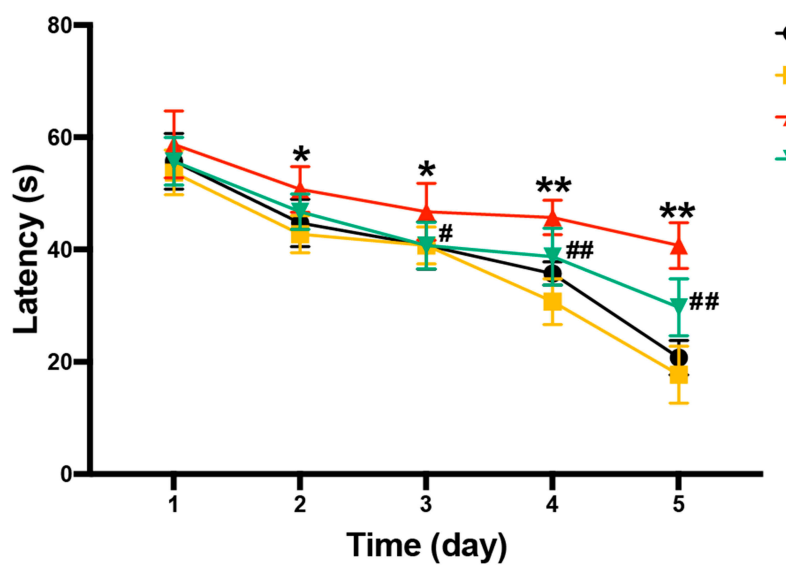

B
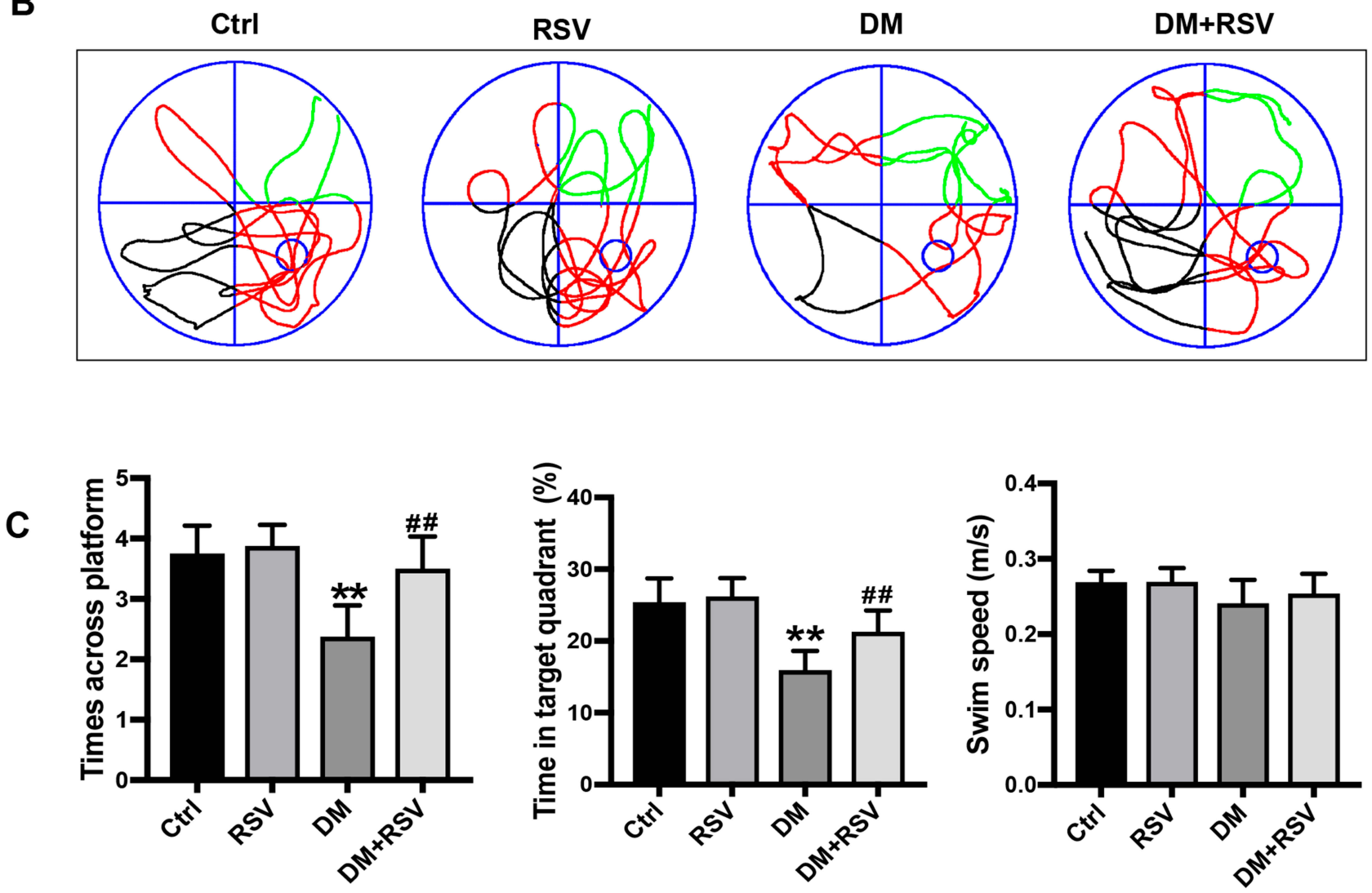

Figure 2 RSV prevents T2DM-induced learning and memory impairment. (A) Evaluation of escape latency in the MWM test to assess spatial information acquisition. (B) Representative traces of swimming in the MWM test after the platform was removed. (C) Swimming speed analysis to determine whether motor function affected the results. Memory capacity is indicated by the number of times the mice crossed the target quadrant and the percentage of time spent in the target quadrant after platform removal on day 6. The data are expressed as the mean \pm SD $(n=8)$. $* P<0.05,{ }^{* * P} P<0.01$ vs $C$ trl; ${ }^{\#} P<0.05,{ }^{\#} P<0.01 v s$. DM.

Abbreviation: MWM, Morris water maze.

Immunofluorescence staining of $\mathrm{Nrf} 2$ was used to investigate the nuclear accumulation of Nrf2 in hippocampal neurons. Compared to the control group, the RSV group had significantly increased Nrf2 nuclear localization. Nrf2 expression was reduced in both the cytoplasm and the nucleus in the DM group compared with the control group. However, the nuclear localization of $\mathrm{Nrf} 2$ was almost normal in the DM + RSV group (Figure 6C). 


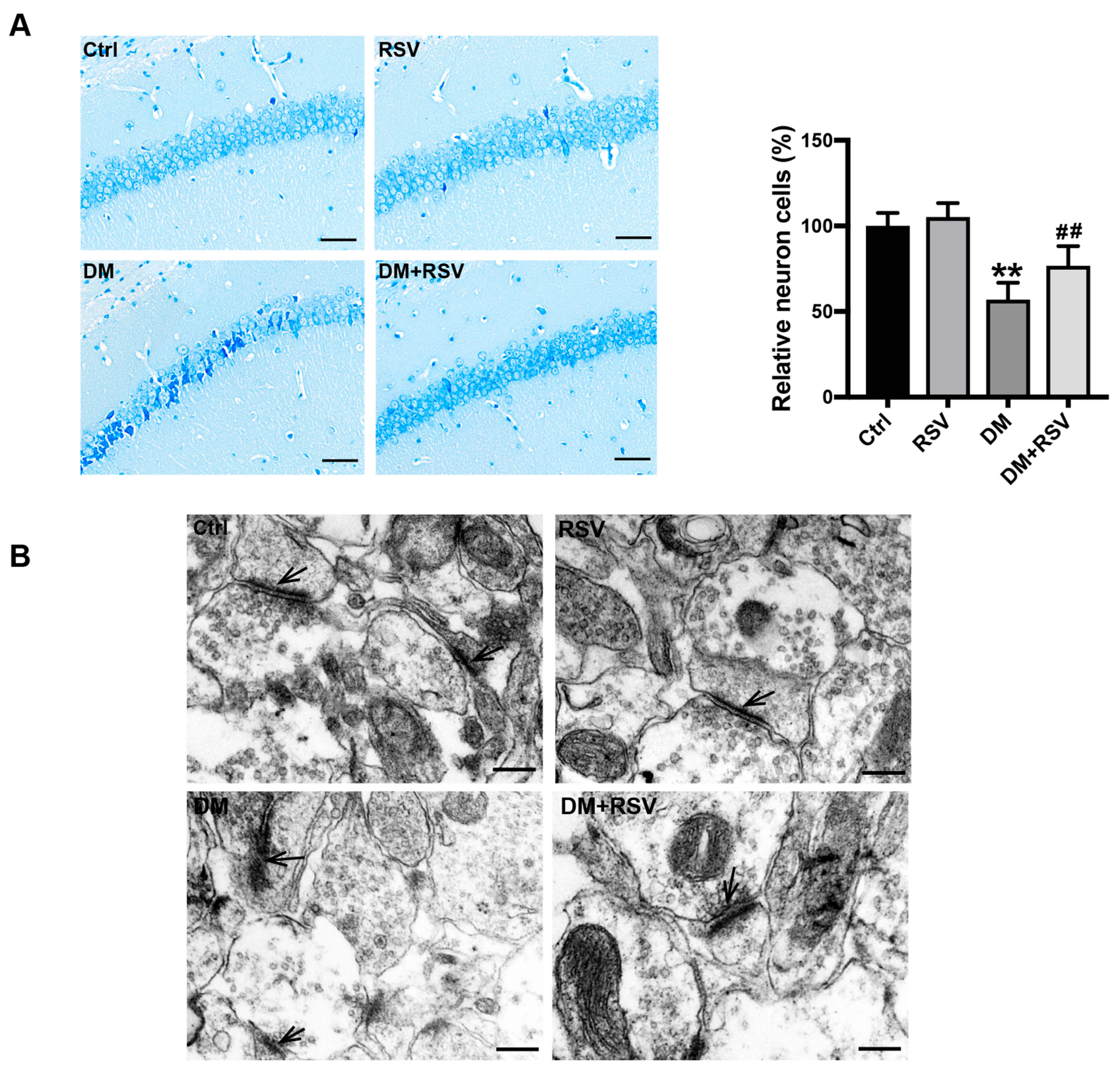

Figure 3 RSV prevents T2DM-induced hippocampal neuronal destruction and synaptic ultrastructural damage. (A) Representative images of Nissl-stained neurons in the mouse hippocampal CAI region $(400 \times$, scale bar $=50 \mu \mathrm{m})$. The number of neurons was quantitatively analyzed. (B) Ultrastructural changes in synapses (indicated by black arrows) in the CAI region of the hippocampus as observed by TEM (Scale bar $=250 \mathrm{~nm})$. The data are expressed as the mean $\pm S D(n=3)$. ${ }^{* * P}<0.01$ vs Ctrl; ${ }^{\#} P<0.0$ Ivs. $D M$.

Abbreviation: TEM, transmission electron microscopy.

\section{RSV Upregulates the Expression of Nrf2-Downstream Antioxidant Target Genes in the Hippocampus}

We confirmed the transcriptional effect of RSV treatment by detecting the levels of the Nrf2 downstream antioxidant genes. Compared to the control mice, the mRNA expressions of NQO-1, $H O-1$, and $M T$ were significantly increased in the RSV group, while were significantly decreased in the DM group. Meanwhile, the mRNA expression of these target genes in the DM + RSV group was significantly increased compared to that in the DM group (Figure 7A). Consistent with the mRNA expression results, the Western blot results showed that the protein levels of NQO1, HO-1, and MT were significantly increased in the RSV-treated control and DM mice (Figure 7B and C). Moreover, SOD and CAT activities were increased significantly in the RSV group and decreased significantly in the DM group compared with the control group. The activities of SOD and 
A

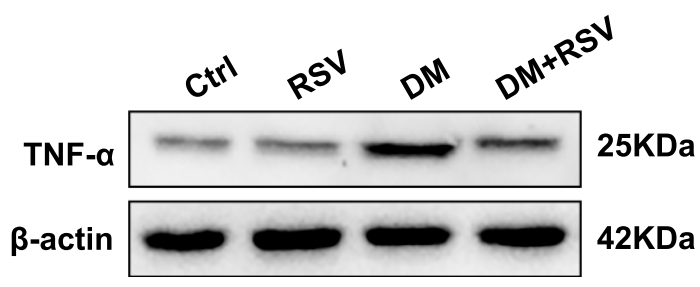

B

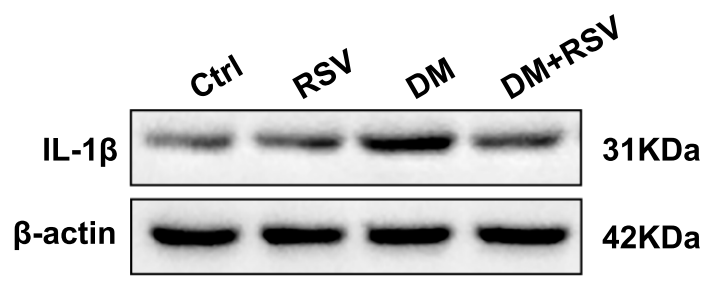

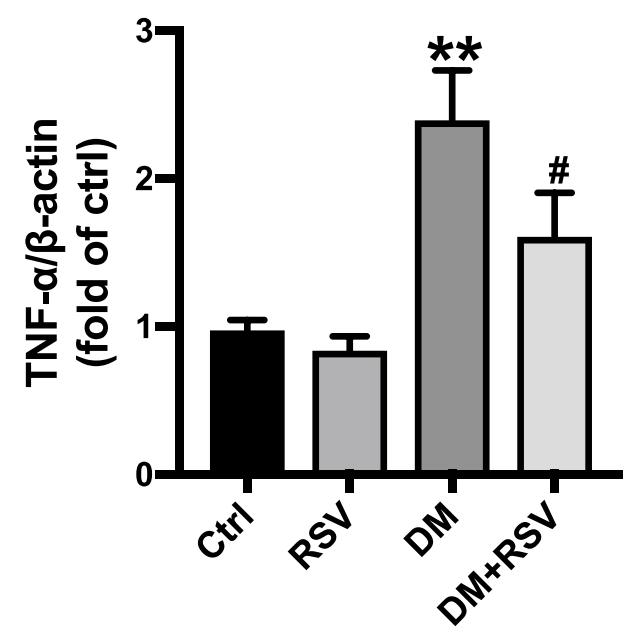

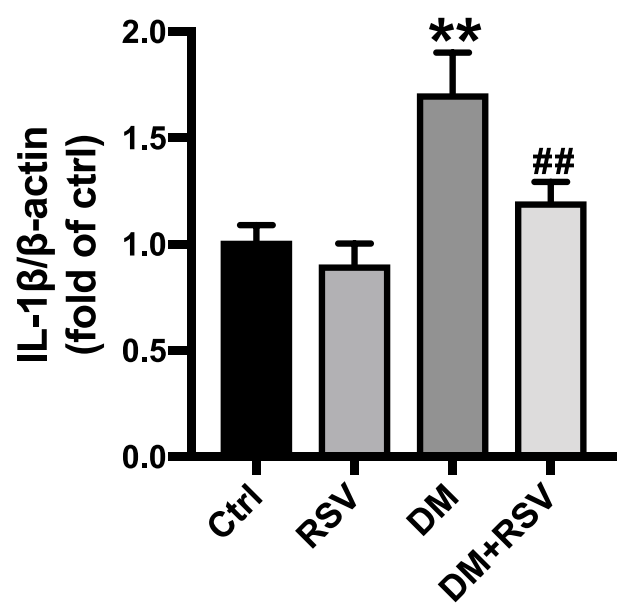

Figure 4 RSV prevents T2DM-induced hippocampal inflammation. (A, B) The levels of the inflammatory factors TNF- $\alpha$ (A) and IL-I $\beta$ (B) were measured by Western blotting. The data are expressed as the mean $\pm S D(n=3)$. ${ }^{* *} P<0.01$ vs $C$ trl; ${ }^{\#} P<0.05,{ }^{\# \#} P<0.0$ Ivs. DM.

Abbreviations: TNF- $\alpha$, tumor necrosis factor- $\alpha$; IL-I $\beta$, interleukin-I $\beta$.

CAT in the DM + RSV group were between those in the control group and those in the DM group (Figure 7D and E). All of the data suggest that RSV may prevent T2DMinduced cognitive impairment by upregulating $\mathrm{Nrf2}$ and its downstream antioxidant genes.

\section{Discussion}

T2DM-induced cognitive impairment not only seriously affects the quality of life of patients but also adds to the economic burden of the family and society due to high medical expenses. With the aging of the population and the increasing prevalence of diabetes, T2DM-induced cognitive impairment not only a medical problem but also a public social issue that needs to be solved urgently. Inflammation and oxidative stress are thought to be important mechanisms of cognitive decline. $^{9,10}$ Therefore, the development of appropriate strategies to prevent hippocampal inflammation and oxidative stress in patients with T2DM is an essential approach to prevent cognitive impairment.

Research shows that ROS levels in diabetic patients are abnormally high, while the expression of antioxidant enzymes is reduced. ${ }^{30-33}$ The severe long-term complications associated with diabetes are tissue damage due to this extremely high glucose level and subsequent oxidative stress. ${ }^{34,35}$ However, individual antioxidants do not provide significant benefits for diabetic complications because they provide only the simple function of ROS scavenging. ${ }^{36}$ Since some synthetic antioxidants, such as triterpenoid derivatives, may cause cardiovascular toxicity, the use of these antioxidants to prevent diabetic nephropathy was urgently stopped in Phase III clinical trials. ${ }^{37}$ As a result, natural antioxidants have been extensively studied for their convenient access and controllable side effects. 
A
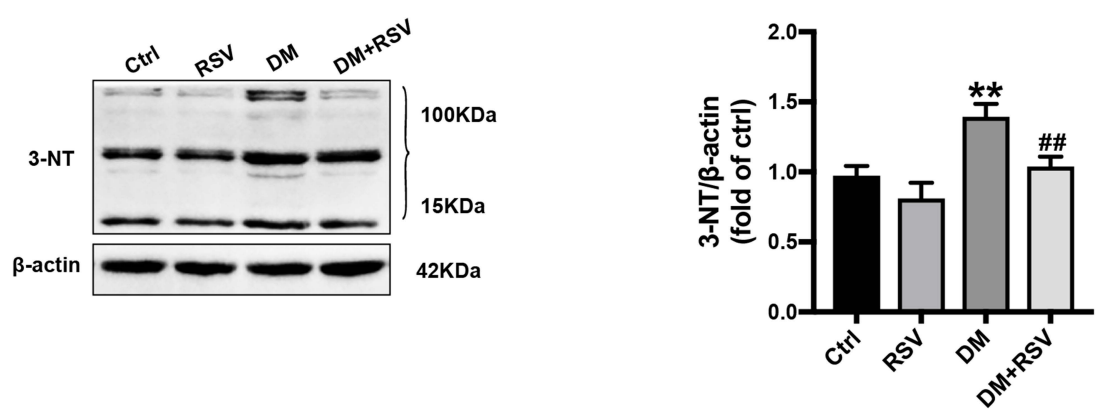

B

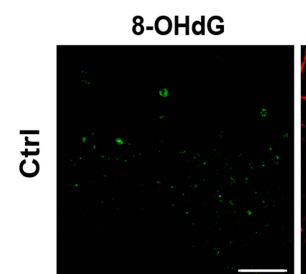

GFAP

DAPI

Merged
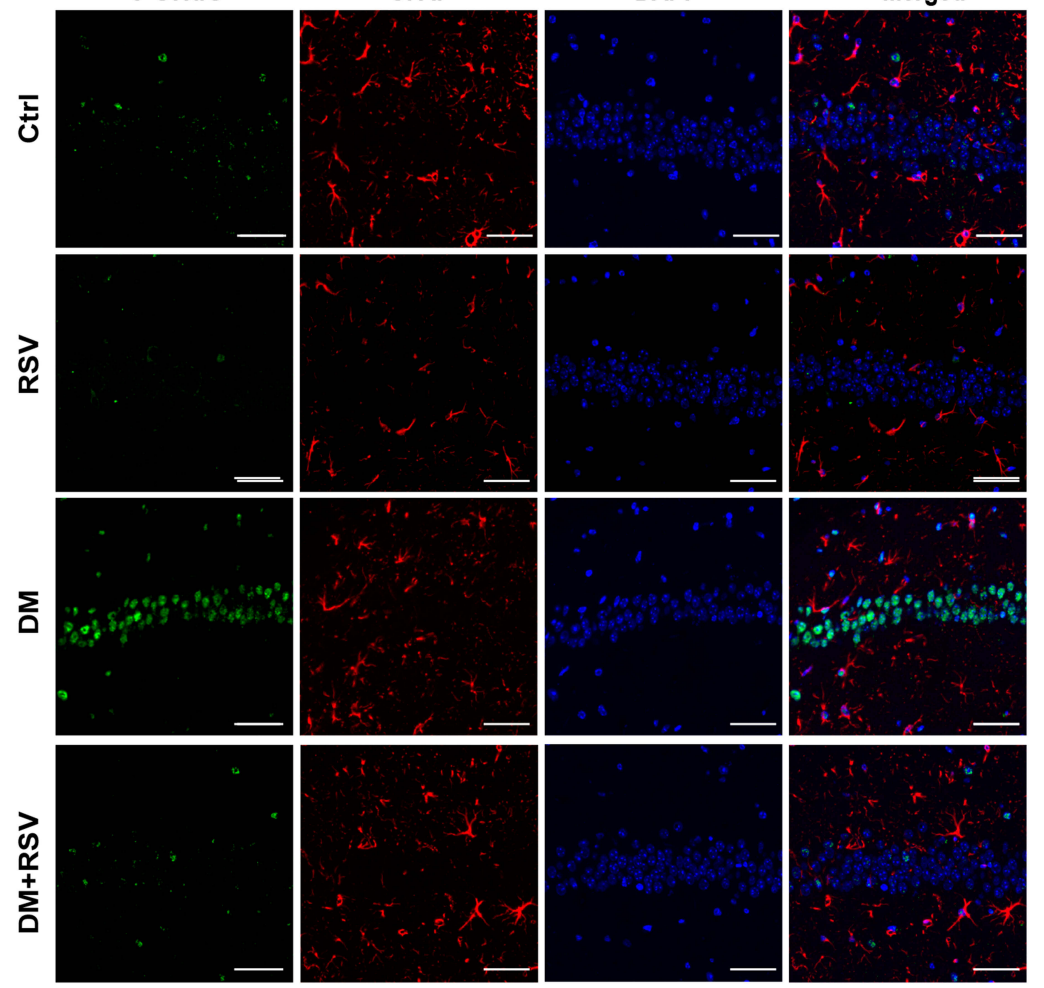

C

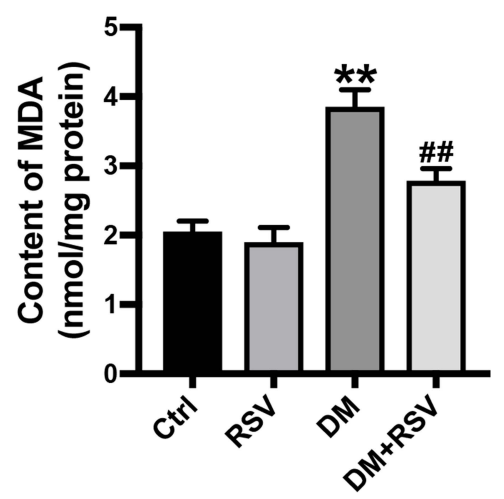

Figure 5 RSV prevents T2DM-induced hippocampal oxidative stress. (A) Detection of 3-NT expression, an indicator of protein nitrification, in the hippocampus by Western blotting to assess oxidative damage. (B) Micrographs of coimmunolabelling with the DNA oxidation markers 8-OHdG (green), GFAP (red) and DAPI (blue) in the hippocampus. The mice hippocampus showed 8-OHdG (green) immunolabeling in neurons (DAPI, blue) and some glial (GFAP, red) cells (Scale bar=I0 $\mu$ m). (C) The determination of malondialdehyde (MDA) content reflected the degree of lipid peroxidation in the hippocampus, and indirectly reflected the degree of neuron damage. The data are expressed as the mean \pm SD $(\mathrm{n}=3)$. **P $<0.01$ vs Ctrl; ${ }^{\#} P<0.0$ Ivs. DM.

Abbreviations: 3-NT, 3-nitrotyrosine; 8-OHdG, 8-hydroxyguanosine; GFAP, glial fibrillary acidic protein; DAPI, 4.6-diamidino-2-phenylindole; MDA, malondialdehyde. 
A

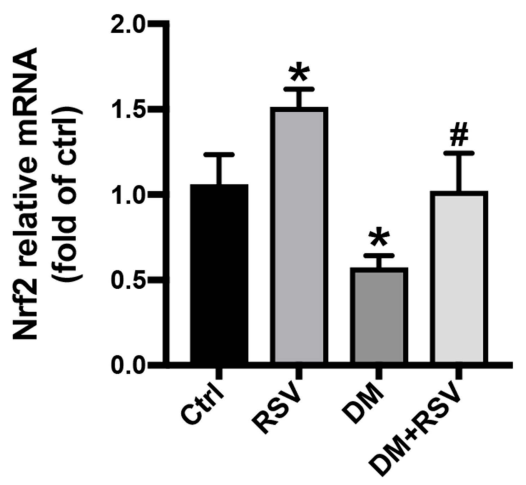

B
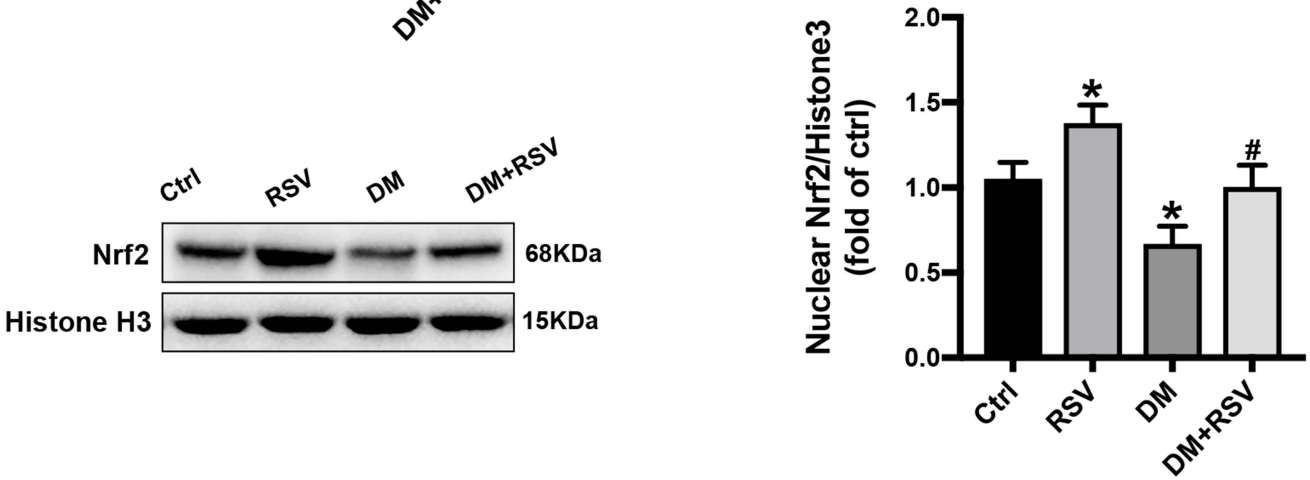

C
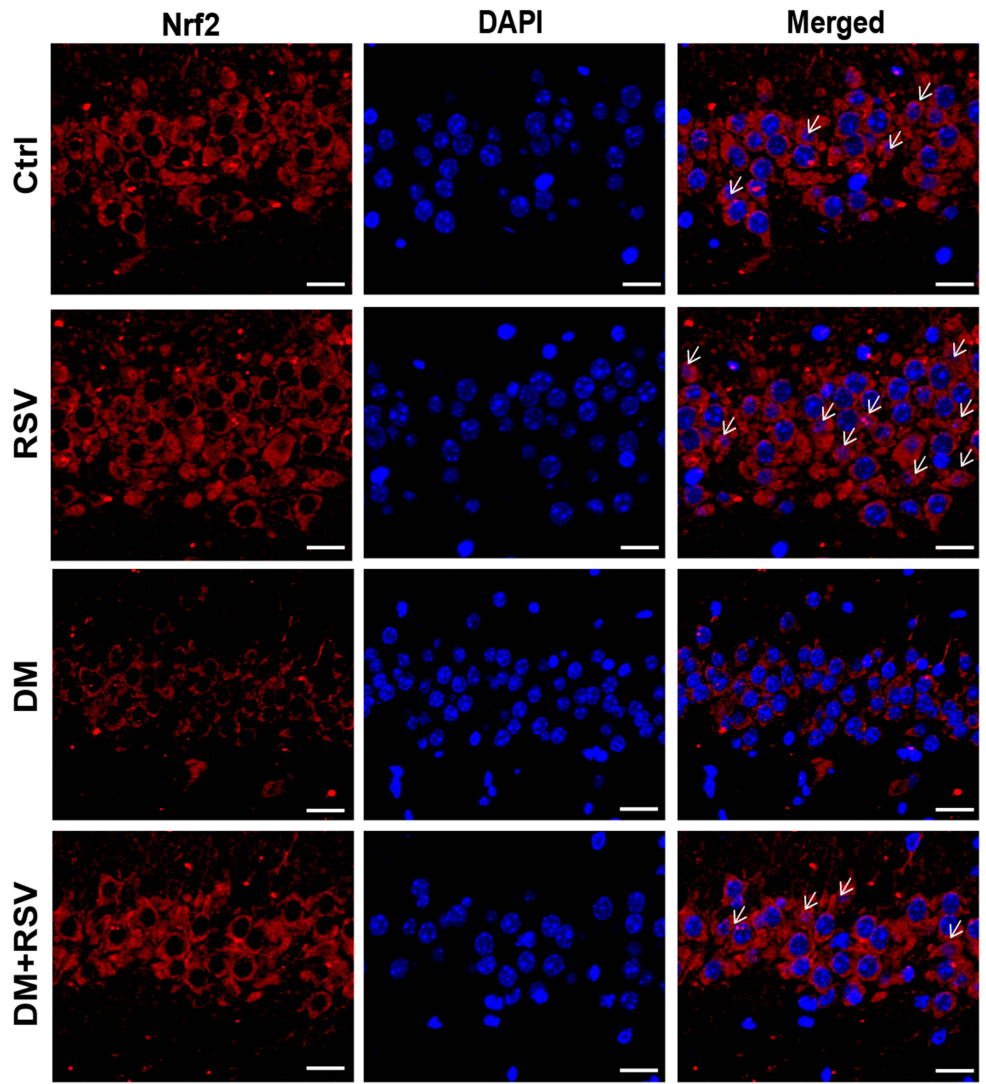

Figure 6 RSV upregulates Nrf2 expression in the hippocampus. (A) The mRNA level of Nrf2 in the hippocampus of mice was measured by qRT-PCR. (B) The expression of Nrf2 nuclear protein in the hippocampus was determined by Western blot. (C) The activation of Nrf2 was analyzed by the immunofluorescence staining of hippocampal tissue sections. Nrf2 (red), nuclear staining by DAPI (blue), and nuclear accumulation (indicated by white arrows). The merged image shows the nuclear localization of the Nrf2 protein (scale bar $=20 \mu \mathrm{m})$. The data are expressed as the mean \pm SD $(n=3) . * P<0.05$ vs Ctrl; ${ }^{\#} P<0.05$ vs DM.

Abbreviations: qRT-PCR, quantitative real-time PCR; DAPI, 4,6-diamidino-2-phenylindole. 
A

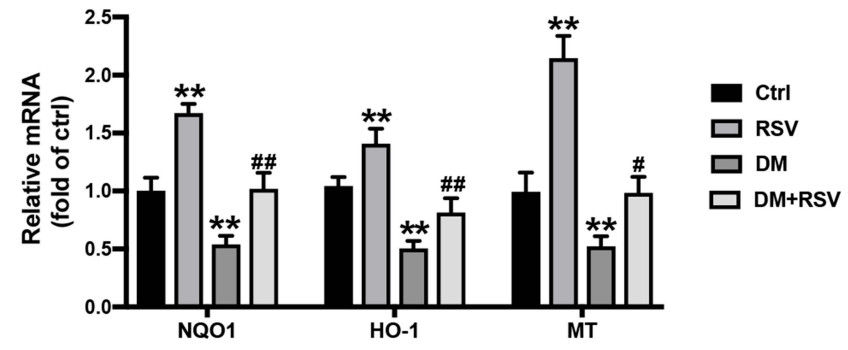

B
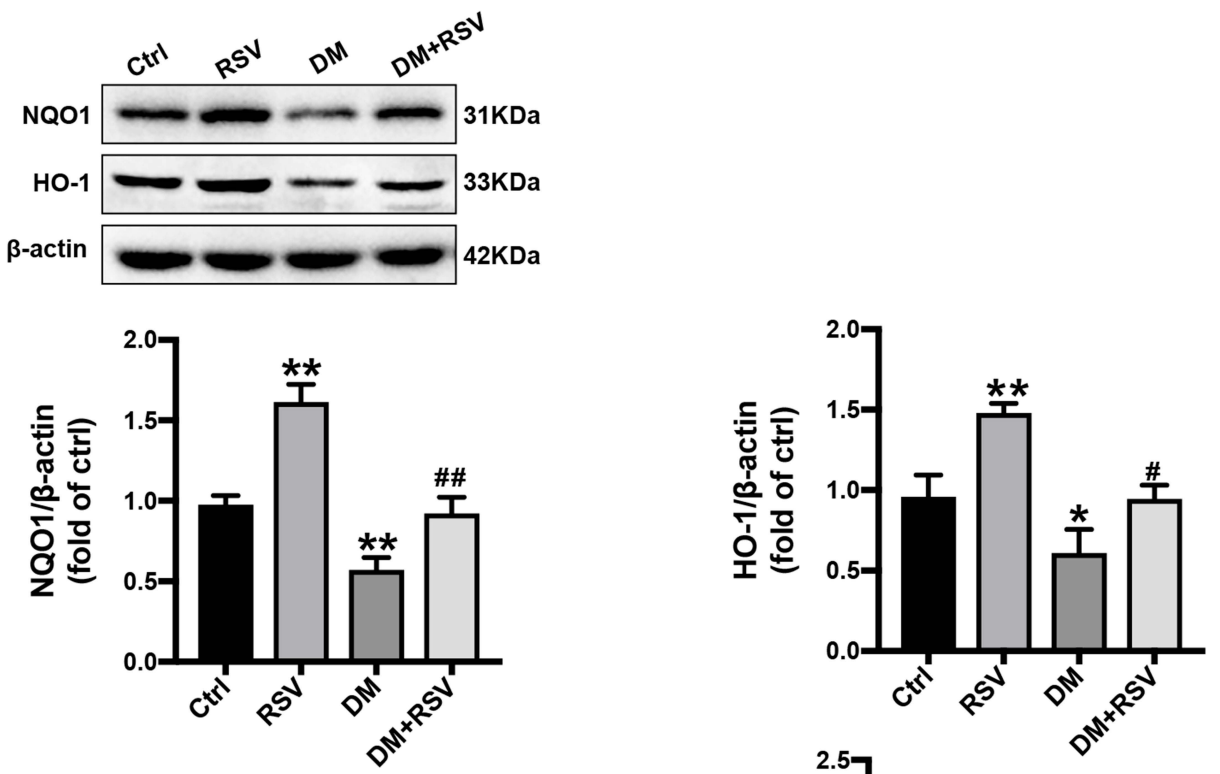

C
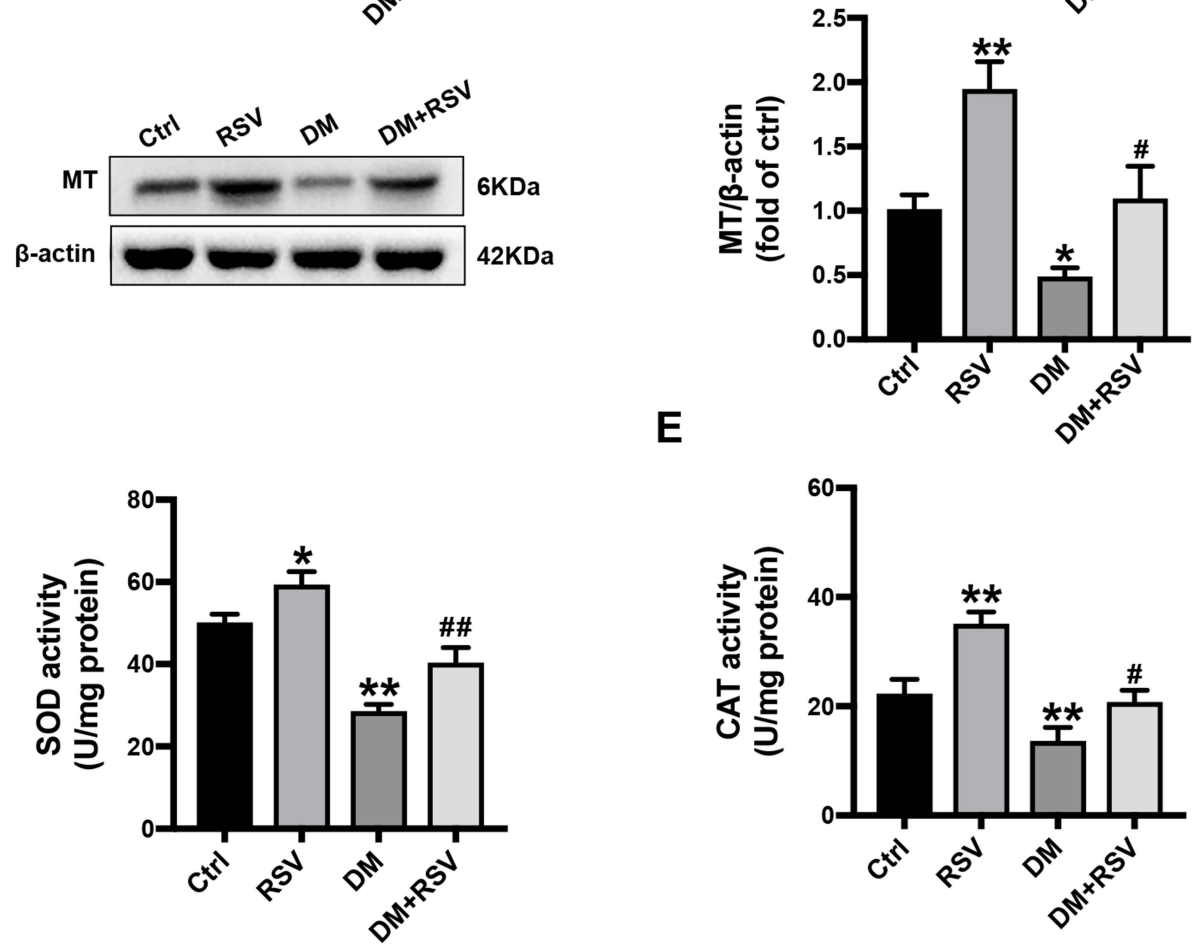

Figure 7 RSV upregulates the expression of Nrf2-downstream antioxidant target genes in the hippocampus. (A) The mRNA levels of the Nrf2 downstream genes NQOI, HO-I, and MT were measured by qRT-PCR. (B, C) The protein levels of NQO-I, HO-I, and MT in the hippocampus were measured by Western blot. (D, E) The activities of SOD (D) and CAT (E) in the hippocampus were detected by the quantitative kit. The data are expressed as the mean \pm SD $(n=3)$. ${ }^{*} P<0.05$, $* * P<0.01$ vs $C$ trl; ${ }^{\#} P<0.05$, ${ }^{\#} P<0.0$ Ivs. DM.

Abbreviations: HO-I, heme oxygenase-I; NQOI, NAD $(\mathrm{P}) \mathrm{H}$, quinone oxidoreductase; MT, metallothionein; SOD, superoxide dismutase; CAT, catalase. 
As a well-known phenolic compound and antioxidant, RSV prevents oxidative damage under diverse pathological situations by controlling inflammation and maintaining the redox homeostasis of cells. ${ }^{38,39}$ Some studies have used RSV to reverse the cognitive deficits caused by diabetes in rats. ${ }^{40,41}$ Consistent with these findings, our study showed that 4 months of RSV treatment significantly reduced the escape latency of T2DM mice and increased the number of crossings and time spent in the target quadrant (Figure 2A-C). In addition, RSV significantly improved T2DM-induced hippocampal neuronal cell damage and synaptic ultrastructure damage (Figure 3A and B). Moreover, RSV significantly prevented the increase of the T2DM-induced inflammatory factors IL- $1 \beta$ and TNF- $\alpha$, which is consistent with previous reports indicating that RSV controls inflammation (Figure 4A and B). Furthermore, RSV prevented the overexpression of the T2DM-induced protein nitration index 3-NT, the DNA oxidation damage index $8-\mathrm{OHdG}$, and the lipid peroxidation end product MDA (Figure $5 \mathrm{~A}-\mathrm{C}$ ). These pieces of evidence predict that RSV improves the cognitive function of T2DM mice, possibly by reducing inflammation and oxidative stress.

$\mathrm{Nrf} 2$ is one of the main factors that protects and restores cell homeostasis. If the activity of $\mathrm{Nrf2}$ is not sufficient to resist oxidative stress or eliminate misfolded and aggregated proteins, it is often related to the etiology and consequences of diabetes. As a sensitive antioxidant gene, Nrf2 expression should increase to resist oxidative stress when excessive ROS is produced in a high glucose environment. However, the expression and activity of $\mathrm{Nrf2}$ in this study were significantly reduced in the 4th month of T2DM induction. Previous studies found a slight increase in Nrf2 expression or activity in the 3rd month in a DMinduced cardiomyopathy model of oxidative damage. However, the expression and effect of Nrf2 were significantly reduced after 6 months of hyperglycemia. ${ }^{42}$ The mechanism by which Nrf2 expression levels increase in the early stage and decrease in the later stage in the T2DM model is not clear. Taken together, the results of previous research and this experiment suggest that the following factors may be involved: (1) the mice in this experiment were exposed to T2DM for too long (4 months); (2) decreased Nrf2 expression is associated with reduced agerelated neural stem cell function; ${ }^{43}$ and (3) the compensatory response of Nrf2 to oxidative stress also decreases with age. ${ }^{44}$ The decrease in Nrf2 expression may be related to the increased proteasome activity of tissues or cells in a high-glucose environment. ${ }^{45}$ Therefore, we assume that elevated levels of Nrf2 in the early phases of T2DM are a compensatory response to T2DM-induced oxidative stress, and sustained oxidative stress will damage the effect of Nrf2, thereby exaggerating the oxidative damage to the hippocampus in the later stages and affecting cognitive function.

In this study, Nrf2 expression was significantly increased in RSV-treated diabetic and nondiabetic mice by mRNA, Western blot, and immunofluorescence analyses (Figure 6A-C). These results suggest that RSV upregulated Nrf2 and promoted Nrf2 nuclear translocation to play a protective role. To further verify the transcriptional function of $\mathrm{Nrf} 2$, downstream antioxidant genes were measured. The expression levels of NQO1, HO-1, and MT in T2DM mice were consistent with that of Nrf2; they were all significantly decreased in the DM group compared with the control group. In addition, the activities of SOD and CAT in the T2DM group were significantly reduced. After RSV treatment, the expression of these antioxidant genes in T2DM mice increased significantly, almost reaching normal levels (Figure 7A-E). All the above data suggest that RSV may upregulate the expression of $\mathrm{Nrf2}$ and downstream antioxidants, thereby preventing T2DMinduced cognitive impairments. In addition to the mechanism of RSV-mediated Nrf2 explained above, there may be other mechanisms that play a role in the prevention of T2DM-induced cognitive impairment, which deserves further discussion. At present, some studies have shown that RSV can effectively prevent damage to various target organs caused by diabetes without inducing a significant

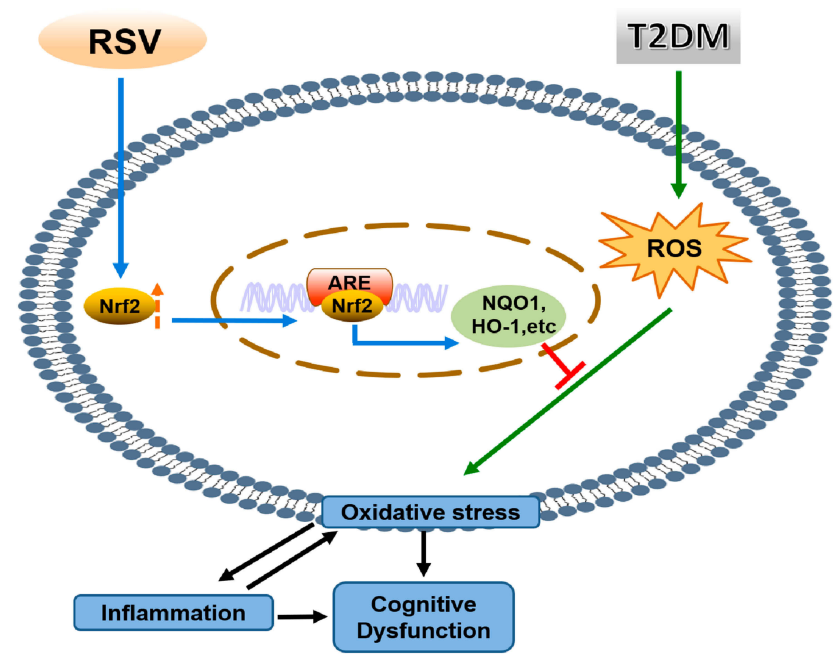

Figure 8 Mechanism diagram. 
hypoglycemic effect. ${ }^{26,27}$ In this study, we did not find a significant difference in blood glucose levels between the RSV-treated T2DM group and the untreated T2DM group (Figure 1E). Therefore, we speculate that the RSVmediated prevention of T2DM-induced cognitive impairment and damage to other organs is not significantly related to its effect on blood glucose. However, whether Nrf2 plays an indispensable role in the neuroprotective effect of RSV remains unknown. These problems need to be further explored in $N r f 2$ knockout mice.

\section{Conclusion}

In summary, we found that RSV prevents T2DM-induced cognitive impairment through anti-inflammatory and antioxidative activities. This effect may be due to the upregulation of Nrf2 expression and downstream antioxidant genes by RSV. The schematic in Figure 8 illustrates the mechanism by which RSV prevents T2DM-induced cognitive impairment.

\section{Acknowledgments}

All authors thank Guangyao Song (Hebei General Hospital) for his help in technical support. This work was funded by the Natural Science Foundation of Hebei Province (Grant No. H2015105083) and the Graduate Innovation Fund of Hebei (Grant No. CXZZBS2019125).

\section{Disclosure}

The authors report that there are no potential conflicts of interest associated with this article.

\section{References}

1. Guariguata L, DR W, Hambleton I, Beagley J, Linnenkamp U, Shaw JE. Global estimates of diabetes prevalence for 2013 and projections for 2035. Diabetes Res Clin Pract. 2014;103(2):137-149. doi:10.1016/j.diabres.2013.11.002

2. Biessels GJ, Koffeman A, Scheltens P. Diabetes and cognitive impairment. J Neurol. 2005;253(4):477-482. doi:10.1007/s00415-005-0036-4

3. Mehla J, Chauhan BC, Chauhan NB. Experimental induction of type 2 diabetes in aging-accelerated mice triggered Alzheimer-like pathology and memory deficits. $J$ Alzheimers Dis. 2014;39(1):145-162. doi:10.3233/JAD-131238

4. Yates KF, Sweat V, Yau PL, Turchiano MM, Convit A. Impact of metabolic syndrome on cognition and brain: a selected review of the literature. Arterioscler Thromb Vasc Biol. 2012;32(9):2060-2067. doi:10.1161/ATVBAHA.112.252759

5. McEwen BS, Magariñ os AḾ, Reagan LP. Studies of hormone action in the hippocampal formation possible relevance to depression and diabetes. J Psychosom Res. 2002;53:883-890. doi:10.1016/S0022-3999(02)00307-0

6. Zhen YF, Zhang J, Liu XY, et al. Low BDNF is associated with cognitive deficits in patients with type 2 diabetes. Psychopharmacology. 2012;227 (1):93-100. doi:10.1007/s00213-012-2942-3

7. Yang Y, Fang H, Xu G, et al. Liraglutide improves cognitive impairment via the AMPK and PI3K/Akt signaling pathways in type 2 diabetic rats. Mol Med Rep. 2018;18:2449-2457.
8. Fang H, Xu X-Y, Xu R-Z, Zhen YF, Xu G, Y-k L. Decreased serum undercarboxylated osteocalcin is associated with cognitive impairment in male patients with type 2 diabetes. J Diabetes Complications. 2018;32(1):56-60. doi:10.1016/j.jdiacomp.2017.09.004

9. Biessels GJ, Strachan MWJ, Visseren FLJ, Kappelle LJ, Whitmer RA. Dementia and cognitive decline in type 2 diabetes and prediabetic stages: towards targeted interventions. Lancet Diabetes Endocrinol. 2014;2(3):246-255. doi:10.1016/S2213-8587(13)70088-3

10. Swomley AM, Butterfield DA. Oxidative stress in Alzheimer disease and mild cognitive impairment: evidence from human data provided by redox proteomics. Arch Toxicol. 2015;89(10):1669-1680. doi:10.1007/ s00204-015-1556-Z

11. Zhang DD, Hannink M. Distinct cysteine residues in Keap1 are required for Keap1-dependent ubiquitination of Nrf2 and for stabilization of Nrf2 by chemopreventive agents and oxidative stress. Mol Cell Biol. 2003;23(22):8137-8151. doi:10.1128/MCB.23.22.81378151.2003

12. Tousoulis D, Papageorgiou N, Androulakis E, et al. Diabetes mellitus-associated vascular impairment: novel circulating biomarkers and therapeutic approaches. J Am Coll Cardiol. 2013;62 (8):667-676. doi:10.1016/j.jacc.2013.03.089

13. Tapias V, Jainuddin S, Ahuja M, et al. Benfotiamine treatment activates the Nrf2/ARE pathway and is neuroprotective in a transgenic mouse model of tauopathy. Hum Mol Genet. 2018;27(16):2874-2892. doi:10.1093/hmg/ddy201

14. Yang Y, Fan C, Wang B, et al. Pterostilbene attenuates high glucose-induced oxidative injury in hippocampal neuronal cells by activating nuclear factor erythroid 2-related factor 2. BBA Mol Basis Dis. 2017;1863(4):827-837. doi:10.1016/j.bbadis.2017.01.005

15. Hosakote YM, Jantzi PD, Esham DL, et al. Viral-mediated inhibition of antioxidant enzymes contributes to the pathogenesis of severe respiratory syncytial virus bronchiolitis. Am J Respir Crit Care Med. 2011;183(11):1550-1560. doi:10.1164/rccm.201010-1755OC

16. Chen M-L, Yi L, Jin X, et al. Resveratrol attenuates vascular endothelial inflammation by inducing autophagy through the cAMP signaling pathway. Autophagy. 2014;9(12):2033-2045. doi:10.4161/ auto. 26336

17. Zeng K, Wang Y, Yang N, et al. Resveratrol inhibits diabetic-induced muller cells apoptosis through microRNA-29b/specificity protein 1 pathway. Mol Neurobiol. 2017;54(6):4000-4014. doi:10.1007/s12035016-9972-5

18. Lee SM, Yang H, Tartar DM, et al. Prevention and treatment of diabetes with resveratrol in a non-obese mouse model of type 1 diabetes. Diabetologia. 2011;54(5):1136-1146. doi:10.1007/s00125-011-2064-1

19. Jimenez-Gomez Y, Mattison JA, Pearson KJ, et al. Resveratrol improves adipose insulin signaling and reduces the inflammatory response in adipose tissue of rhesus monkeys on high-fat, high-sugar diet. Cell Metab. 2013;18(4):533-545. doi:10.1016/j. cmet.2013.09.004

20. Khazaei M, Karimi J, Sheikh N, et al. Effects of resveratrol on receptor for advanced glycation end products (rage) expression and oxidative stress in the liver of rats with type 2 diabetes. Phytother Res. 2016;30(1):66-71. doi:10.1002/ptr.5501

21. Kim YH, Kim YS, Kang SS, Cho GJ, Choi WS. Resveratrol inhibits neuronal apoptosis and elevated $\mathrm{Ca} 2+/$ calmodulin-dependent protein kinase II activity in diabetic mouse retina. Diabetes. 2010;59 (7):1825-1835. doi:10.2337/db09-1431

22. Ma X, Sun Z, Liu Y, Jia Y, Zhang B, Zhang J. Resveratrol improves cognition and reduces oxidative stress in rats with vascular dementia. Neural Regen Res. 2013;8(22):2050-2059. doi:10.3969/j.issn.16735374.2013.22.004

23. Chatterjee S, Khunti K, Davies MJ. Type 2 diabetes. Lancet. 2017;389(10085):2239-2251. doi:10.1016/S0140-6736(17)30058-2

24. Walle T, Hsieh F, DeLegge MH, Oatis JE, Walle UK. High absorption but very low bioavailability of oral resveratrol in humans. Drug Metab Dispos. 2004;32(12):1377-1382. doi:10.1124/dmd.104.000885 
25. Wenzel E, Soldo T, Erbersdobler H, Somoza V. Bioactivity and metabolism of trans-resveratrol orally administered to Wistar rats. Nutr Food Res. 2005;49:482-494. doi:10.1002/mnfr.200500003

26. Wang G, Song X, Zhao L, Li Z, Liu B. Resveratrol prevents diabetic cardiomyopathy by increasing Nrf2 expression and transcriptional activity. Biomed Res Int. 2018;2018:2150218.

27. Zhao Y, Song W, Wang Z, et al. Resveratrol attenuates testicular apoptosis in type 1 diabetic mice: role of Akt-mediated Nrf2 activation and p62-dependent Keap1 degradation. Redox Biol. 2018;14:609-617. doi:10.1016/j.redox.2017.11.007

28. Boocock DJ, Faust GES, Patel KR, et al. Phase I dose escalation pharmacokinetic study in healthy volunteers of resveratrol, a potential cancer chemopreventive agent. Cancer Epidemiol Biomarkers Prev. 2007;16(6):1246-1252. doi:10.1158/1055-9965.EPI-07-0022

29. Morris RG, Garrud P, Rawlins J, O'Keefe J. Place navigation impaired in rats with hippocampal lesions. Nature. 1982;297 (5868):681. doi:10.1038/297681a0

30. Zhang H, Davies KJA, Forman HJ. Oxidative stress response and Nrf2 signaling in aging. Free Radic Biol Med. $2015 ; 88(\mathrm{Pt}$ B):314-336. doi:10.1016/j.freeradbiomed.2015.05.036

31. Vanessa Fiorentino T, Prioletta A, Zuo P, Folli F. Hyperglycemiainduced oxidative stress and its role in diabetes mellitus related cardiovascular diseases. Curr Pharm Des. 2013;19(32):5695-5703. doi:10.2174/1381612811319320005

32. Jha JC, Banal C, Chow BS, Cooper ME, Jandeleit-Dahm K. Diabetes and kidney disease: role of oxidative stress. Antioxid Redox Signal. 2016;25(12):657-684. doi:10.1089/ars.2016.6664

33. Newsholme P, Cruzat VF, Keane KN, Carlessi R, de Bittencourt PI. Molecular mechanisms of ROS production and oxidative stress in diabetes. Biochem J. 2016;473(24):4527-4550. doi:10.1042/BCJ20160 $503 \mathrm{C}$

34. Brownlee M. Biochemistry and molecular cell biology of diabetic complications. Nature. 2001;414(6865):813. doi:10.1038/414813a

35. Sheetz MJ, King GL. Molecular understanding of hyperglycemia's adverse effects for diabetic complications. JAMA. 2002;288 (20):2579-2588. doi:10.1001/jama.288.20.2579

36. Jung KA, Kwak MK. The Nrf2 system as a potential target for the development of indirect antioxidants. Molecules. 2010;15 (10):7266-7291. doi:10.3390/molecules 15107266
37. de Zeeuw D, Akizawa T, Audhya P, et al. Bardoxolone methyl in type 2 diabetes and stage 4 chronic kidney disease. $N$ Engl J Med. 2013;369(26):2492-2503. doi:10.1056/NEJMoa1306033

38. Banerjee B, Nandi P, Chakraborty S, Raha S, Sen PC, Jana K. Resveratrol ameliorates benzo(a)pyrene-induced testicular dysfunction and apoptosis: involvement of p38 MAPK/ATF2/iNOS signaling. J Nutr Biochem. 2016;34:17-29. doi:10.1016/j.jnutbio. 2016.04.003

39. González-Rodríguez Á, Santamaría B, Mas-Gutierrez JA, et al. Resveratrol treatment restores peripheral insulin sensitivity in diabetic mice in a sirt1-independent manner. Mol Nutr Food Res. 2015;59(8):1431-1442. doi:10.1002/mnfr.201400933

40. Schmatz R, Mazzanti CM, Spanevello R, et al. Resveratrol prevents memory deficits and the increase in acetylcholinesterase activity in streptozotocin-induced diabetic rats. Eur J Pharmacol. 2009;610(1-3):42-48. doi:10.1016/j.ejphar.2009.03.032

41. Gocmez SS, Şahin TD, Yazir Y, et al. Resveratrol prevents cognitive deficits by attenuating oxidative damage and inflammation in rat model of streptozotocin diabetes induced vascular dementia. Physiol Behav. 2019;201:198-207. doi:10.1016/j.physbeh.2018.12.012

42. Bai Y, Cui W, Xin Y, et al. Prevention by sulforaphane of diabetic cardiomyopathy is associated with up-regulation of Nrf2 expression and transcription activation. J Mol Cell Cardiol. 2013;57:82-95. doi:10.1016/j.yjmcc.2013.01.008

43. Tan Y, Ichikawa T, Li J, et al. Diabetic downregulation of Nrf2 activity via ERK contributes to oxidative stress-induced insulin resistance in cardiac cells in vitro and in vivo. Diabetes. 2011;60 (2):625-633. doi:10.2337/db10-1164

44. Corenblum MJ, Ray S, Remley QW, et al. Reduced Nrf2 expression mediates the decline in neural stem cell function during a critical middle-age period. Aging Cell. 2016;15(4):725-736. doi:10.1111/ acel. 12482

45. Luo ZF, Qi W, Feng B, et al. Prevention of diabetic nephropathy in rats through enhanced renal antioxidative capacity by inhibition of the proteasome. Life Sci. 2011;88(11-12):512-520. doi:10.1016/j. lfs. 2010.12 .023

\section{Publish your work in this journal}

Diabetes, Metabolic Syndrome and Obesity: Targets and Therapy is an international, peer-reviewed open-access journal committed to the rapid publication of the latest laboratory and clinical findings in the fields of diabetes, metabolic syndrome and obesity research. Original research, review, case reports, hypothesis formation, expert opinion and commentaries are all considered for publication. The manuscript management system is completely online and includes a very quick and fair peer-review system, which is all easy to use. Visit http://www.dovepress.com/testimonials.php to read real quotes from published authors. 